\section{Pre-operative radiographs for dental implants - are selection criteria being followed?}

\author{
S. J. J. McCrea ${ }^{1}$
}

VERIFIABLE CPD PAPER

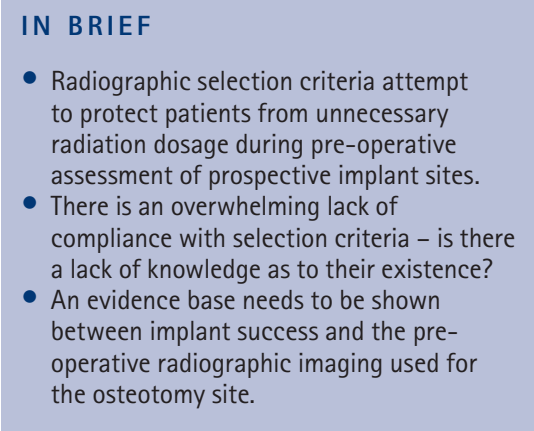

- Radiographic selection criteria attempt to protect patients from unnecessary radiation dosage during pre-operative assessment of prospective implant sites.

There is an overwhelming lack of compliance with selection criteria - is there a lack of knowledge as to their existence?

An evidence base needs to be shown between implant success and the prethe osteotomy site.

\begin{abstract}
Objectives To determine by anonymous survey whether the active membership of a particular dental society with variable training and clinical experience in dental implant insertion and restoration followed the published selection criteria for the pre-operative radiographic assessment of prospective dental implant osteotomy sites. Design Postal survey. Subjects and methods A postal questionnaire was sent to all professionally active members of the British Society of Periodontology $(n=560)$ during July 2005. The mailing was repeated in September 2005. Results Four hundred and fifty-nine questionnaires were returned a response rate of 81.5\%. One hundred and seventy-nine (39\%) respondents were not involved in placing or restoring dental implants and were excluded. Of the remaining $280(61 \%), 15(5 \%)$ clinicians limited themselves to placing implants, 85 (30\%) limited themselves to restoring implants, while 181 (65\%) both placed and restored their own implants. Two hundred and twenty-eight clinicians (80.8\%) did not follow UK selection criteria for single sites; 217 clinicians (77.5\%) did not follow the criteria for multiple sites. Two hundred and sixty-three clinicians (94\%) did not follow USA selection criteria. There were no statistically significant differences in compliance based on clinical experience or the number of implants placed. The only statistically significant difference was as a result of training those clinicians who had attended formal training courses were more likely to comply with published selection criteria. Significant differences also existed between the groups in the specific cross-sectional imaging modalities chosen. Conclusion Over 80\% of respondents are not following the UK or USA selection criteria for pre-implant imaging assessment. The results of this survey call into question the clinical relevance, usefulness, or knowledge of existing selection criteria. There is a need for new selection criteria developed by both radiologists and experienced implant clinicians.
\end{abstract}

\section{INTRODUCTION}

The replacement of missing teeth with implant retained fixed or removable appliances is now well-documented. The high success rate of osseointegrated dental implants has led to their use as a common clinical protocol to re-establish oral health in the edentulous and partially edentulous subject. ${ }^{1-4}$

It is well-accepted that the recipient bed for a dental implant should be routinely assessed by clinical and visual examination and finally by radiographic

IImplantologist and Dental Surgeon, Clinical Director, The Dental Implant and Gingival-Plastic Surgery Centre, 717 Christchurch Road, Bournemouth, BH7 6AF

Correspondence to: Dr Shane McCrea

Email: shanemccrea@aol.com

\section{Refereed Paper}

Accepted 28 January 2008

DOI: $10.1038 /$ sj.bdj.2008.524

${ }^{\circledR}$ British Dental Journal 2008; 204: 675-682 analysis. Respected researchers ${ }^{5}$ have stressed that the indications for the most frequently used imaging modalities in implant dentistry should be placed on clinical need and biological risk to the patient. The radiographic assessment should be accurate enough to include the various anatomic structures, the presence of abnormalities and disease, the morphology of the implant site and information regarding bone density, such that the placement of the implant can be carried out with confidence. ${ }^{6}$ The analysis of prospective implant sites by radiographic means has led to the formulation of 'selection criteria.'

To our knowledge, and as a result of our literature search, no studies have been published in which compliance with selection criteria for the radiographic assessment of prospective implant sites has come under scrutiny. This is the first study that systematically confronts the issue of radiographic compliance in dental implant planning.

Selection criteria have been formulated to address the growing need for the protection of the patient from unnecessary radiation dosage during the treatment planning phase for dental implants. ${ }^{7,8}$ As shown in Table 1, the effective doses from various implant imaging modalities varies enormously from very low dose periapicals to relatively high dose CT scans. As a result it is not surprising that radiographic 'selection criteria' for pre-operative assessment of potential implant sites have been published in recent years in both the UK and the US.

The Faculty of General Dental Practice (UK)'s publication Selection criteria for dental radiography $(2004)^{7}$ quotes two tables from the consensus document of the European Association for Osseointegration, Guidelines for the use of diagnostic imaging in implant dentistry. ${ }^{18}$ 


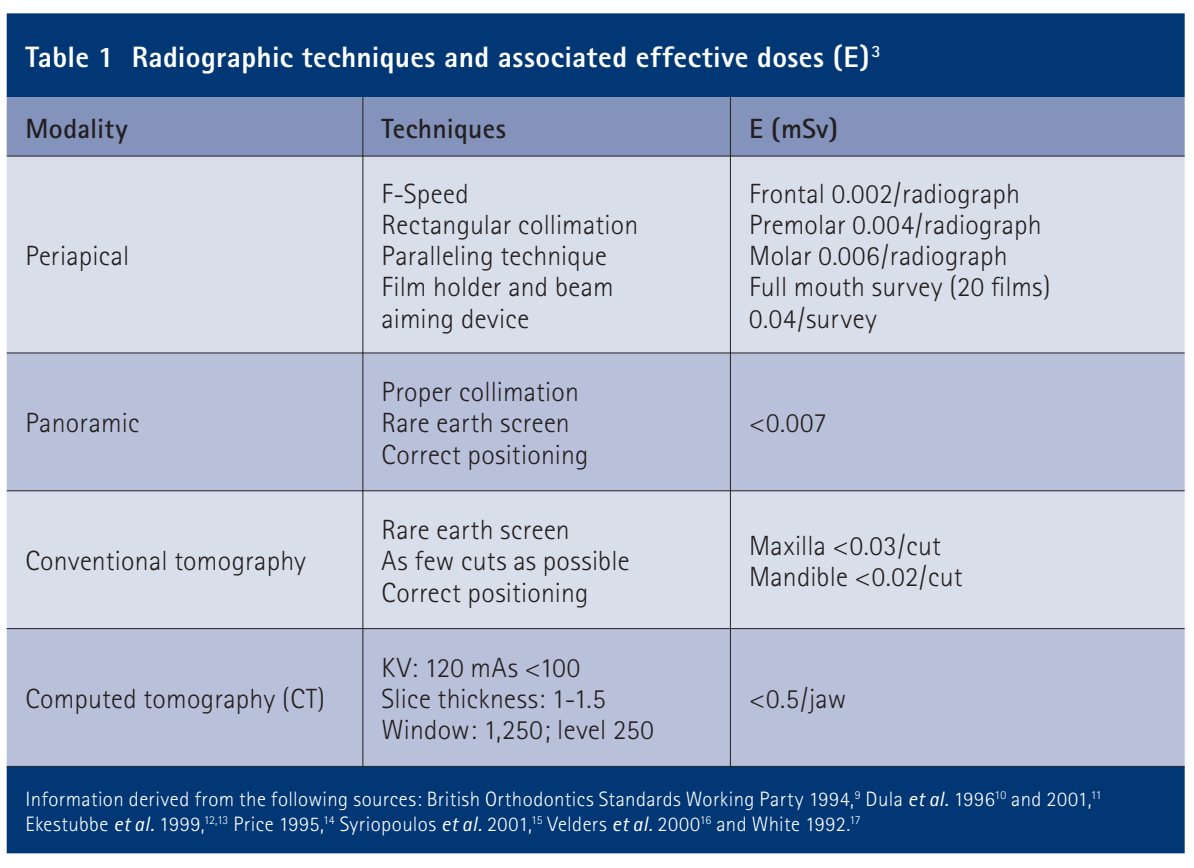

Table 2 Summary of FGDP(UK) radiographic selection criteria for dental implant planning?

1. Clinical and visual examination including palpation and study model assessment.

2. An initial intra-oral two-dimensional periapical radiograph for single site assessment.

3. A panoramic radiograph for multiple implant sites - this being dependent on dose implications and the wish to investigate anatomical factors.

4. The use of cross-sectional imaging will be dependent on the individual case and on the availability of the resource, the preferred choice being computed tomography (CT).

Table 3 Summary of AAOMR radiographic selection criteria for dental implant planning ${ }^{8}$

1. Clinical and visual examination including palpation and study model assessment.

2. A two-dimensional radiographic examination of either panoramic and/or periapical films.

3. In all cases a cross-sectional image of the site, using preferentially, conventional tomography.

By recommending the use of these two tables, the FGDP(UK) advocates radiographic imaging that will be dependant on the number of proposed implant sites. The summary of their recommendations can be found in Table 2 .

The American Academy of Oral and Maxillofacial Radiology $(\mathrm{AAOMR})^{8}$ has a different approach; their recommendations can be found in Table 3 .

\section{MATERIALS AND METHODS}

A questionnaire, having closed questions with single-response tick-box options, was designed. Before distribution, the questionnaire was piloted on an implant study group (the Wessex Dental Implantology Society, having a membership of 70 , all with varying degrees of implant experience), thus allowing refinements and amendments to the questionnaire. The questionnaire was divided into ten distinct sections which were used to determine:

1. The respondants' experience with implants - the number that are placed and/or restored per year

2. Their numbers of years of clinical experience

3. Their training - formal or otherwise

4. Their usual methods of clinical and placing and/or restoring dental radiographic assessment of single and multiple potential implant sites

5. Finally, the respondents were asked about the availability of more advanced two- and three-dimensional imaging modalities.

Using the information derived from these distinct areas of questioning it was believed that relationships could be demonstrated between years of experience and numbers of implants being placed and/or restored, and further, that surgical expertise and training may have a distinct bearing on the type of radiographic modalities being employed. Finally, the information collected would allow a systematic analysis of the groups of clinicians who do or do not comply with the published selection criteria recommendations.

The membership list of the British Society of Periodontology (BSP) at the time of this survey comprised 630, which included 70 members who were no longer professionally active, either through retirement or because they were deceased. The 560 active members of the BSP were selected to take part in the postal survey. A questionnaire (Appendix 1) was sent out in July 2005 together with a detailed covering letter, which explained the background of the survey, and a reply paid envelope. Reminder letters were mailed to non-responders in September 2005.

\section{RESULTS}

Four hundred and fifty-nine questionnaires were returned - a response rate of $81.5 \%$. One hundred and seventy-nine (39\%) respondents were not involved in placing or restoring dental implants and were excluded. Of the remaining 280 (61\%), 15 (5\%) clinicians limited themselves to placing implants, 85 (30\%) limited themselves to restoring implants, while 181 (65\%) both placed and restored their own implants. Thus, three 'implant activity' groups were recognised for analysis. The number of clinicians limiting themselves to placing implants was small - being only 15 (5\%) - and so their results, though recorded and 'analysed', were considered as being of interest but not of statistical worth as a stand-alone group. 
1. The results of the survey were analysed in two steps:

2. Firstly, to display any trends that could be seen on an intra-group level

3. Secondly, for any significant intergroup relationships or differences to be demonstrated.

\section{The number of implants placed and/or restored per year by each group}

There was considerable variation in the average number of implants placed and/ or restored per year by each group, as shown in Figure 1.

\section{Clinicians limited to placing implants}

Of the 15 clinicians who limited themselves to placing implants, five (33.3\%) placed less than 20 per year, three (20\%) placed between 21 and 50, three (20\%) placed between 51 and 99, and four (26.7\%) clinicians placed more than 100 implants per year.

\section{Clinicians limited to restoring implants}

Of the 85 clinicians who only restored implants, $52(61.17 \%)$ restored less than 20 per year, $23(27.05 \%)$ restored between 21-50, six (7.06\%) restored between 51 and 99 and only four (4.71\%) clinicians restored more than 100 implants per year.

\section{Clinicians both placing and restoring their implants}

Of the 181 clinicians who both placed and restored implants, 47 (26\%) dealt with less than 20 per year, 49 (27\%) placed and restored between 21-50, 33 (18.2\%) placed and restored between 51 and 99 and 52 (28.8\%) clinicians placed and restored more than 100 implants per year.

\section{Number of years of experience of the clinicians when compared to the number of implants being placed/restored (Fig. 2)}

There was also considerable variation in the number of years experience the clinicians had in the different groups. However, as was to be expected, those clinicians placing and/or restoring over 100 implants per year were those with the longest experience.

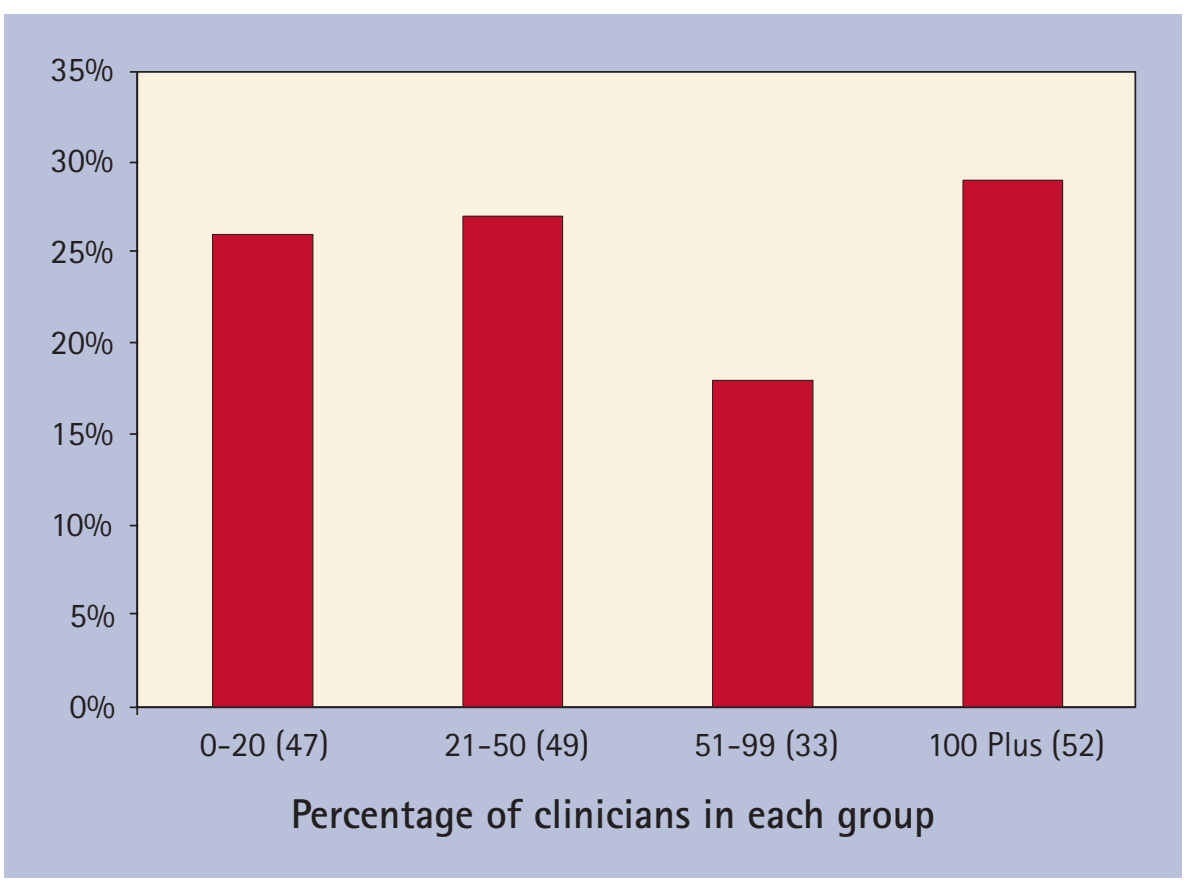

Fig. 1 Yearly implant activity of the three groups of respondents

\section{Clinicians limited to placing implants}

In Figure 2, the relationship between the years of experience and the number of implants being placed is very clearly displayed. All of the group placing 100 or more implants have 10 years or more experience in placing implants. It is safe to state that the progressive increase in surgical experience of these clinicians shows a corresponding increase in the number of implants the clinicians will be placing.

\section{Clinicians limited to restoring}

Though not so well demarcated, there is a direct relationship between the number of implants being restored and the number of years of experience the clinician had. The relationship is statistically significant (chi-square analysis, $\mathrm{p}<0.001$ ) - see Table 4 . Once again, the clinicians with more experience are to be found as the overwhelming majority in the 100-plus group - both the 100plus group and the 51-99 group bringing significant numbers into this group.

\section{Clinicians both placing and restoring implants}

Here, those surgeons with limited experience (0-2 years experience) are to be found only in the two lower bands of clinical activity. Those clinicians with 3-5 years experience show a distinct trend in the number of implants that they place and restore - 32\% in the 0-20 band, 21\% in the 21-50 band, 7\% in the 51-99 band to only $2.5 \%$ in the 100 -plus

\begin{tabular}{|c|c|c|c|c|c|}
\hline \multirow{2}{*}{$\begin{array}{l}\text { Survey } \\
\text { question } 2\end{array}$} & \multicolumn{4}{|c|}{ Survey question 3} & \multirow{2}{*}{ Total } \\
\hline & 1 & 2 & 3 & 4 & \\
\hline 1 & 30 & 29 & 25 & 18 & 102 \\
\hline 2 & 5 & 27 & 18 & 27 & 77 \\
\hline 3 & 0 & 18 & 9 & 15 & 42 \\
\hline 4 & 0 & 2 & 9 & 48 & 59 \\
\hline Total & 35 & 76 & 61 & 108 & 280 \\
\hline
\end{tabular}


band. The overwhelming majority of the 100-plus activity group are those clinicians with 10 or more years experience of implant activity (76\%).

\section{Types of formal training received (Figure 3)}

The questionnaire also sought information on the types of formal training courses clinicians had received. These could have included:

- Masters degree programs on implant dentistry or prosthodontics

- Diploma or certificate programmes

- Other MSc programmes

- Dental implant manufacturers' courses

- Continuing education courses, or any other course.

It was understood right from the onset that the relative infancy of the newer MSc (Implant Dentistry), Diploma in Implant Dentistry and MClinDent (Prothodontics) courses would see a low affirmative response amongst the respondants.

\section{Clinicians limited to restoring implants}

Figure 3 shows the various forms of training received by those clinicians that only restored implants. As can be seen, the majority of clinicians in this group restored 50 or fewer implants per year and almost all of them obtained their training from manufacturers' or general practitioners' day courses. Very few had received formal training in implant rehabilitation therapy.

\section{Clinicians both placing} and restoring implants

Once again the data shows an overwhelming reliance on clinical training being provided by manufacturers' and general practitioners' day training courses, irrespective of the average number of implant cases being treated per year. And again the MSc (Implant Dentistry) and MClinDent formal courses have few responders.

\section{Usual methods of clinical and radiographic assessment of single and multiple potential implant sites (Fig. 4)}

The aim of this part of the study was the evaluation of the type of radiographic

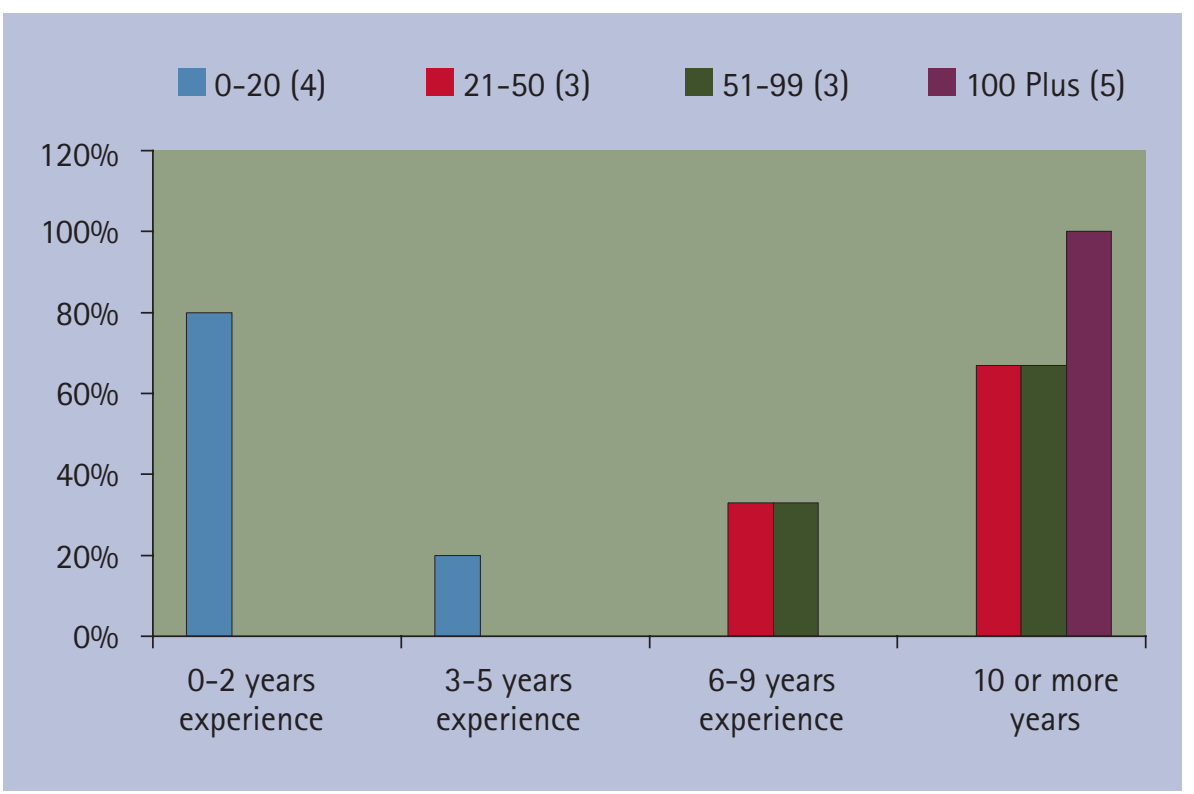

Fig. 2 Relationship between numbers of implants placed and years of experience for the three groups of respondents

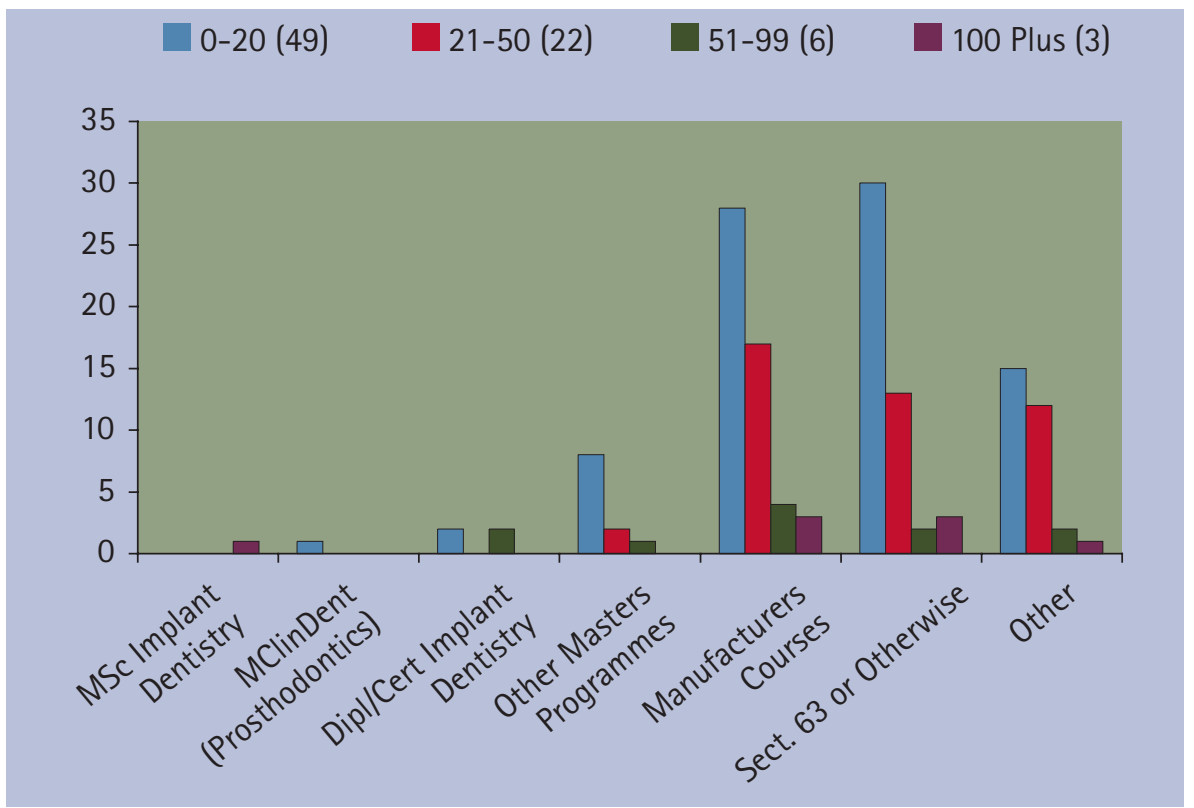

Fig. 3 Types of formal training received by the respondents

examinations clinicians employed when assessing single and multiple implant sites. The data obtained was broken down according to the clinicians' clinical experience and expertise.

\section{Clinicians only restoring implants}

Here the graph is separated clearly into data for single sites and data for multiple sites. The general trend indicates that the greater the number of implants restored, the greater the use of imaging modalities, although cross-sectional imaging is not commonly used. Where single sites are concerned, 100\% of the clinicians restoring more than 100 implants restrict themselves to the use of a periapical radiograph. Further, 100\% of those same clinicians prescribed a dental panoramic tomography (DPT) for the multiple site cases - this is certainly in line with the UK selection criteria recommendations. Those same clinicians then limit themselves as to which periapical radiographs are necessary for further information.

\section{Clinicians both placing and restoring implants}

For those clinicians both placing and restoring implants, much greater emphasis is placed on imaging as a whole. Here 


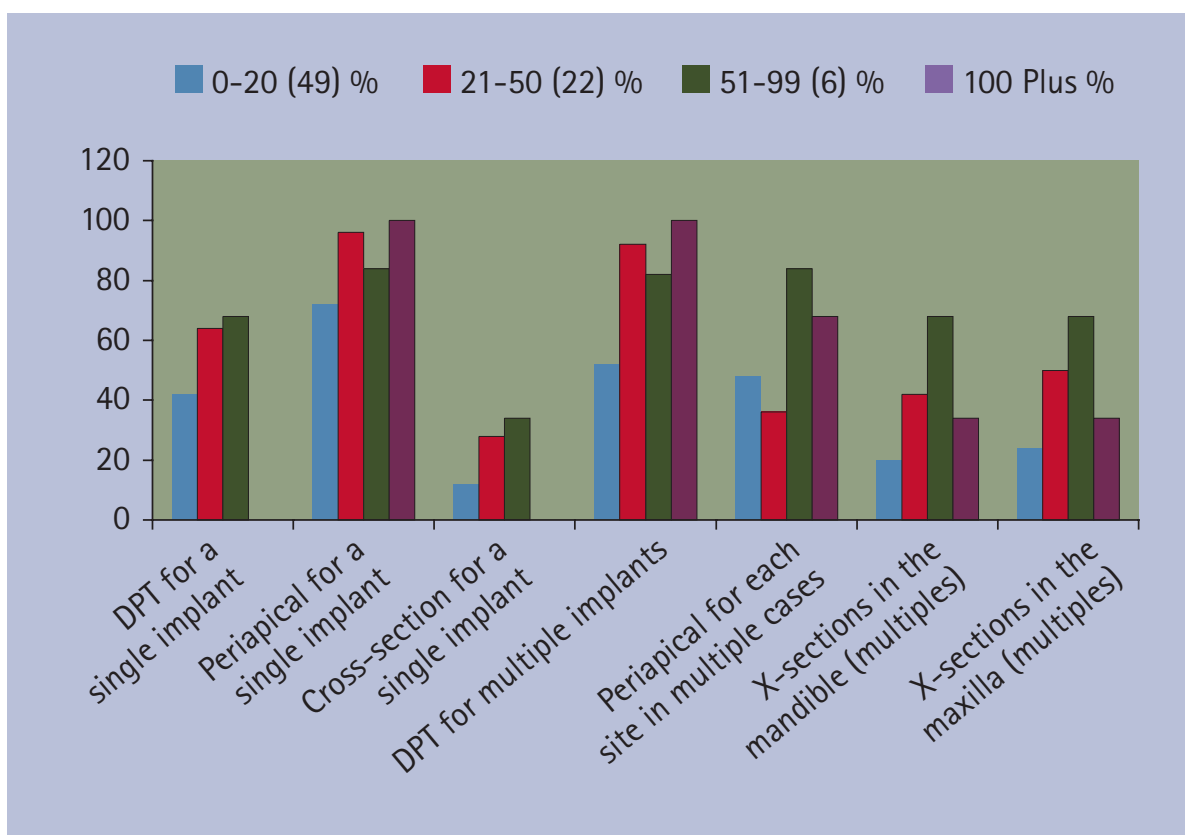

Fig. 4 Respondents' usual methods of clinical and radiographic assessment of potential implant sites

clinical activity appears to make very little difference in the prescribing pattern, with a full range of imaging modalities being used by the overwhelming majority of each activity group. However, cross-sectional imaging remains infrequent for single site assessment, while periapiacals for multiple sites are infrequent - both the latter and former being very much in line with selection criteria recommendations. The group of clinicians only placing implants (15 or $5 \%$ of the respondants) were considered too small to allow meaningful trends to be demonstrated graphically.

\section{Use of cross-sectional imagery}

Further analysis of the types of crosssectional imaging used overall by the clinicians revealed that for those clinicians using cross-sectional imaging:

- Twenty-two (8\%) clinicians used simple linear tomography

- Sixty-nine (25\%) used multidirectional tomography

- One hundred and forty-nine (53\%) used computed tomography (CT)

- Only seven (2.5\%) used cone beam CT

- One $(0.3 \%)$ used magnetic reasonance imaging.

If the USA selection criteria recommendations for cross-sectional imagery of prospective implant sites were being followed, a 100\% result would be expected for conventional tomography. In fact only 33\% of the respondants were using this form of imagery. The results demonstrated that the overwhelming majority of clinicians prescribed CT imaging for cross-sectional investigation, regardless of the 'known' high radiation dose implications.

\section{Statistical analysis}

The results from all three groups of clinicians, including the small group that only placed implants, were tabulated to allow chi-square analysis. The three groups of clinicians were analysed separately for their compliance with the published selection criteria.

- Two hundred and twenty-eight clinicians $(80.8 \%)$ did not follow UK selection criteria for single implant sites

- Two hundred and seventeen clinicians (77.5\%) did not follow UK selection criteria for multiple implant sites

- Two hundred and sixty-three clinicians (94\%) did not follow USA selection criteria recommendations

- There were no statistically significant differences in compliance based on clinical experience ( $p>0.7$ ), or the number of implants being placed ( $p>0.7$ )

- Statistically significant differences were shown to exist as a result of training ( $p<0.05)$ : those clinicians who had attended formal training courses were more likely to comply with published UK selection criteria, whether it was, eg, MSc (Implant Dentistry) or other formal MScs

- Significant differences ( $p<0.05$ ) also existed between the groups in the specific cross-sectional imaging modalities chosen.

\section{DISCUSSION}

The intention of this survey was to determine whether this sample group of active clinicians exhibited compliance with the published selection criteria recommendations for the radiographic analysis of prospective dental implant sites. The results demonstrated that this was not the case: this sample, which was the active membership list of the British Society of Periodontology, overwhelmingly did not follow the published UK or USA selection criteria recommendations. The excellent response rate of $81.5 \%$ to the survey would indicate that the membership considered the questioning in the survey to be of merit and yet, at the same time, the analysis of the responses indicated that the greater majority of the membership did not actively comply with the published recommendations.

A question could then be posed asking whether the selection criteria are actually taken seriously by clinicians; the FGDP(UK) selection criteria have an acknowledged low evidence base, being derived from EAO guidelines (an expert panel) in conjunction with expert opinion of the FGDP panel. The US guidelines have a similarly low level of evidence to support their use.

In this study the survey questions had been divided into key features that we considered could possibly influence the level of compliance with radiographic recommendations amongst the respondents. We felt that logic dictated that the more complicated implant cases would be carried out by the more experienced clinicians; those clinicians would then require the use of more advanced imaging and be more systematic in its usage, thus allowing a higher degree of success. In fact this was not the case: the statistical analysis showed no significant differences in compliance based on clinical experience or the number of implants being placed. 
The well-documented high success rates of osseointegrated dental implants has led to their use as a common clinical protocol to re-establish oral health in the edentulous and partially edentulous. The planning for dental implant insertion relies heavily on the ability to analyse 'unseen' anatomical architecture and as such, the utilisation of radiographic modalities is an integral part of pre-implant assessment. Therefore, the systematic use of radiographic modalities would appear logical for the safe and predictable placement of dental implants. Where single implants are being planned, the recommended imaging is that of the periapical radiograph, ${ }^{19}$ and though there is a preference for the periapical radiograph among the respondents, a very high proportion of clinicians still appear to require the use of a DPT for these same single implant cases - which by their very nature could be described as the 'simple' cases - as demonstrated graphically in Figure 4. Maybe the frequent use of the DPT in these cases is simply a 'safety' measure - it would appear that panoramics are being used as a comparison between the contra-lateral teeth and the anatomical danger zones regardless of their visual discrepancies and the higher dose implications to the patient. These latter points have previously been published in the results of a survey carried out by Sakakura et al. ${ }^{20}$

Where clinicians limit themselves to restoring implants, significant differences $(p<0.05)$ were shown to exist amongst the groups for single implant site assessment for the taking of a panoramic, the use of a magnification factor, and the prescription for cross-sectional imaging. The clinicians who surgically place and then restore their own implants show great similarity in the prescribing profiles in all four of their activity groups, regardless of whether they are experienced or inexperienced. One of the original hypotheses was that clinicians who place more implants would be carrying out more complex treatments and therefore, request more imaging - no visible trend of this is apparent in the graphs. Instead it may well be that with more experience, confidence increases and reliance on imag- ing decreases. This is in opposition to the clinicians who limit themselves to restoring implants.

We hope that perceived surgical necessity has determined the frequent need for cross-sectional imaging. It is noteworthy that clinicians who prescribe cross-sectional imaging in the mandible for multiple implant site assessment appear to be the same as those prescribing for the maxilla. It is then worrying that there is such a frequent use of CT (53\% of the respondents prescribing cross-sectional imaging) among the respondents. It has been widely recorded by many research$\mathrm{ers}^{21-23}$ that if bone width and lingual undercut can be estimated sufficiently well during clinical examination then a panoramic film is accurate enough to obtain the information regarding implant length. Therefore, this CT frequency could be attributed to the availability of the device in regional hospitals or even over-zealousness, or worse still manufacturer-generated implant drillguidance software. However, should these really be criteria for its usage when the multiple imaging obtained from it is beyond what is actually necessary in much of implant planning? Are patients being subject to higher doses of radiation on the grounds of ease of availability?

The FGDP(UK) are specific in their selection criteria recommendations: '... CT is associated with considerable dose implications that need to be justified, ${ }^{7}$ or as Engelmann et al. ${ }^{24}$ have stated, 'one should expect a substantial increase in the benefit of information provided by CT scanning to justify the risk associated with the dose required'. The justification is hard to warrant amongst the respondents. Hatcher et al. ${ }^{25}$ are open in stating that 'CT imaging has a low value when considering the ratio between diagnostic potential, cost of study, and risk to the patient.'

Clinicians using other cross-sectional imaging such as linear tomography, multi-directional tomography and cone-beam $\mathrm{CT}$ were in the minority - 8\%, 25\% and 2.5\% respectively. Yet all these devices subject the patient to much lower radiation dosage - see Table 1 . The argument then arises that the images obtained from CT are so superior to conventional tomography that this is a good enough reason for its use. If this argument was to hold true then more frequent use could be made of cone beam $\mathrm{CT}$, with its marked reduction in radiation dosage and its comparable accuracy to traditional CT. ${ }^{24,26}$

The lack of compliance with the selection criteria recommendations does raise a question as to whether the clinicians were actually aware of their existence. It may be that radiographic selection criteria are not part of the implant manufacturers' courses and general practitioners' day courses that this survey has shown to be so popular with the respondents. Once again, mention needs to be made of a possible relation between CT scanning and manufacturers' courses for implant positioning/drill guidance software - if this is the case, then this type of course must start to stress whether their techniques are appropriate within the principle of ALARA (as low as reasonably achievable).

Finally, a statistically significant difference was found to exist as a result of training. Those clinicians who had attended formal training courses were more likely to comply with published selection criteria, whether they were, for example, MSc (Implant Dentistry) or other formal MScs. It is fair to postulate that these formal courses which are not manufacturer-generated have taught modules that consider the appropriateness of radiographic imaging in view of their radiation exposure. However, many studies are limited by sample size and ours is no exception. Approximately one third of the sample from the British Society of Periodontology were not involved in implant placement and restoration. More accurate data may have been obtained by choosing a sample group where all the clinicians sampled were involved in implant placement and/or restoration. We believe that further studies should be carried out with a larger sample size of this latter cohort to verify the results/conclusions drawn in this study.

\section{CONCLUSION}

Our present data provides evidence that over $80 \%$ of the respondents to this survey are not following the published UK 
and USA selection criteria recommendations for pre-implant radiographic imaging assessment. The lack of compliance calls into question the respondents' awareness of, and the clinical relevance and usefulness and the lack of supporting evidence for these existing selection criteria.

There is a need for research that can lead to the development of selection criteria that would be taken seriously by clinicians. Such research would certainly have to show a correlation between actual success rates of implants and the radiographic imaging that was used to assess the implant sites pre-operatively.

To Eric Whaites (Director of Dental Radiology, Guys' Hospital, The Dental Institute, King's College London) for his immense help in directing the research for this paper, which was carried out in part fulfilment of my MSc (Dental and Maxillofacial Radiology).

To Ron Wilson, Statistician, King's College London, for his statistical analysis of the collated results.

And to both Ashley and Damian, my sons, for their great interest and assistance in collating the responses to the survey.

1. Adell R. Clinical results of osseointegrated implants supporting fixed prostheses in edentulous jaws. J Prosthet Dent 1983: 50: 251-254.

2. Quirynen $M$, Naert I, van Steenberghe D, Dekeyser C, Callens C. Periodontal aspects of osseointegrated fixtures supporting a partial bridge. An up to 6 -years retrospective study. J Clin Periodontol 1992; 19: 118-126.

3. Karroussis I K, Salvi G E, Heitz-Mayfield L J A,
Bragger U, Hammerle C H F, Lang N P. Long-term implants prognosis in patients with and without a history of chronic periodontitis: a 10-year prospective cohort study of the ITI Dental Implant System. Clin Oral Implants Res 2003; 14: 329-339.

4. Lang N P, Berglundh T, Heitz-Mayfield L J, Pjetursson B E, Salvi G E, Sanz M. Consensus statements and recommended clinical procedures regarding implant survival and complications. Int J Oral Maxillofac Implants 2004; 19(Suppl): 140-149.

5. Bolin A, Eliasson S. Panoramic and tomographic dimensional determinants for maxillary osseointegrated implants. Comparison of the morphological information potential of two and three dimensional radiographic systems. Swed Dent J 1995; 19: $65-71$.

6. Dixon D R, Morgan R, Hollender L G, Roberts FA Neal R B. Clinical application of spiral tomography in anterior implant placement: a case report. J Periodontol 2002; 73: 1202-1209.

7. Pendlebury M E, Horner K, Eaton K A. Selection criteria for dental radiography. London: Faculty of General Dental Practice (UK), 2004.

8. Tyndall A A, Brooks S L. Selection criteria for dental implant site imaging: a position of the American Academy of Oral and Maxillofacial Radiology. Oral Surg Oral Med Oral Pathol Oral Radiol Endod 2002; 89: 630-637.

9. British Orthodontic Standards Working Party. Guidelines for the use of radiographs in clinical orthodontics. London: British Orthodontic Society, 1994.

10. Dula K, Mini R, van der Stelt P F, Lambrecht J T, Schneeberger P, Buser D. Hypothetical mortality risk associated with spiral computed tomography of the maxilla and mandible. Eur J Oral Sci 1996; 104: 503-510.

11. Dula K, Mini R, van der Stelt P F, Buser D. The radiographic assessment of implant patients - decision making criteria. Int J Oral Maxillofac Implants 2001; 16: 80-89.

12. Ekestubbe A. Conventional spiral and low dose computed mandibular tomography in dental implant planning. Goteborg, Sweden: Goteborg University, 1999. Thesis.

13. Ekestubbe A, Grondahl K, Grondahl H G. Quality of preimplant low dose tomography. Oral Surg Oral Med Oral Pathol Oral Radiol Endod 1999; 88: 738-744.
14. Price C. Sensitometric evaluation of a new E-Speed dental radiographic film. Dentomaxillofac Radiol 1995; 24: 30-36.

15. Syriopoulos K, Velders X L, Sanderink G C, van Der Stelt P F. Sensitometric and clinical evaluation of a new F-speed dental X-ray film. Dentomaxillofac Radio/ 2001: 30: 40-44.

16. Velders $X L$, Jacobs R, Gijbels F. Dosimetric evaluation of tomographic techniques. In Jacobs $R$, Gijbels F (eds) Oral Imaging 2000. Final report of the Minosquare concerted action. pp 65-72. Leuven: EDMIN, Department of Periodontology, KU Leuven, 2000. ISBN 90-804618-2-2.

17. White $\mathrm{S} C$. Assessment of radiation risk from dental radiography. Dentomaxillofac Radiol 1992; 21: 118-126.

18. Harris D, Buser D, Dula K et al. European Association for Osseo-integration. Guidelines for the use of diagnostic imaging in implant dentistry. Clin Oral Implants Res 2002; 13: 566-570.

19. Jacobs R. Pre-operative planning for the placement of a single implant. Ned Tijdschr Tandheelkd 1999; 106: 187-190.

20. Sakakura C E, Morais J A, Loffredo L C, Scaf G. A survey of radiographic prescription in dental implant assessment. Dentomaxillofac Radio/ 2003; 32: 397-400.

21. Wyatt C C, Pharoah M J. Imaging techniques and image interpretation for dental implant treatment. Int J Prosthodont 1998; 11: 442-452.

22. Mupparapu $M$, Singer $S R$. Implant imaging for the dentist. J Can Dent Assoc 2004; 70: 32

23. Frei $C$, Buser D, Dula K. Study on the necessity for cross-sectional imaging of the posterior mandible for the treatment planning of standard cases in implant dentistry. Clin Oral Implants Res 2004; 15: 490-497.

24. Engelmann M J, Sorenson J A, Moy P. Optimum placement of osseo-integrated implants. J Prosthet Dent 1998; 59: 467-473.

25. Hatcher D C, Dial C, Mayorga C. Cone-beam CT for pre-surgical assessment of implant sites. J Calif Dent Assoc 2003; 31: 825-833.

26. Scarfe W C. Farman A G, Sukovic P. Clinical applications of cone-beam computed tomography in dental practice. J Can Dent Assoc 2006; 72: 75-80.

\section{Appendix 1 The survey questionnaire}

Radiographic assessment of prospective dental implant sites

A postal questionnaire survey

Your response to this questionnaire is confidential and anonymous

1. Do you place dental implants?

Do you restore dental implants?
Yes / No (please circle)

Yes / No (please circle)

2. How many implants do you place/restore on average in any one year? (please tick)
0 -20 implants per year
21-50 implants per year
51-99 implants per year
100 implants or more per year

3. For how many years have you been placing implants or restoring implants? (please tick)
0 -2 years
3-5 years
6-9 years
10 or more years 


\section{RESEARCH}

\section{Appendix 1 The survey questionnaire}

\section{Continued from page 681}

4. What sort of formal training have you had for the surgical placement or restoration of implants? (please tick - you may tick more than one, if appropriate)

$\square$ MSc (Implant Dentistry)

Please specify the Institution:

$\square$ MClinDent (Prosthodontics)

Please specify the Institution:

$\square$ Diploma/Certificate in Implant Dentistry

Please specify the Institution:

$\square$ Other Masters programmes

Please specify the Institution:

$\square$ Courses arranged by dental implant manufacturers

$\square$ Privately run courses, whether Section 63 or otherwise

The following questions are to determine whether you have a preference for a particular implant 'system' or a cross-over in techniques:

5. Do you restrict yourself to ONE implant system?

Yes / No (please circle)

6. When assessing for a SINGLE implant, do you:
Take an OPG?
Yes/No
Take a periapical of the site?

Yes / No

Use ball-bearing analysis or other radio-opaque

measurement method for the occluso-apical assessment? Yes / No

Apply ridge mapping for the alveolar width? Yes / No

ALWAYS prescribe cross-sectional imagery? Yes / No

7. When assessing for MULTIPLE implants, do you:
Take an OPG?
Yes / No

Take periapical views of EACH site?

Yes / No

Use ball-bearing analysis or other radio-opaque

measurement method for the occluso-apical assessment? Yes / No

Apply ridge mapping for the alveolar width at each site? Yes / No

ALWAYS prescribe cross-sectional imagery:
in the maxilla?
Yes / No
in the mandible?
Yes/No

8. Is the cross-sectional imagery as asked in questions 6 and 7 :
a) Scanora type?
Yes/No
b) Computed tomography?
Yes / No

9. Do you have an OPG unit in your practice?

Yes / No

If the answer is YES:

10. Are there cross-sectional facilities on the OPG unit?

Yes / No 